\title{
Laser Beam Shaping Using Hollow Optical Fiber and Its Application in Laser Induced Thermal Printing
}

\author{
Jonghoon $\mathrm{Yi}^{*}$, Kangin Lee, Illhyun Park, and Jin Hyuk Kwon \\ Department of Physics, Yeungnam University, Kyungsan 712-749, Korea
}

(Received December 1, 2008 : accepted December 19, 2008)

\begin{abstract}
A Gaussian beam of single mode fiber laser was changed into a ring-shaped pattern after it was transmitted through the hollow optical fiber. The ring-shaped beam was focused on a plane by an $f-\theta$ lens and it was scanned by a Galvano-mirror. The spatial profile of laser energy incident on a plane had two peaks at both sides of the scanned linear track. The profile was compared with the result obtained when the Gaussian beam was dithered transversely by an acousto-optic modulator. It is found that hollow optical fiber beam shaper can replace acousto-optic beam dithering device which is employed in a laser induced thermal printing system.
\end{abstract}

Keywords: Beam shaping, Hollow optical fiber, Laser thermal printing

OCIS codes : (110.6820) Thermal imaging; (140.3300) Laser beam shaping; (310.6860) Thin films optical properties

\section{INTRODUCTION}

In the flat panel displays industry, lasers are finding applications in several manufacturing processes such as laser annealing of silicon to a microcrystalline state, [1] laser ablation of indium tin oxide for patterning, [2] and laser induced thermal printing (LITI) of color filter or organic material [3]. LITI is known as the more applicable method than inkjet or thermal evaporation methods for large area, high resolution OLED (organic light emitting diode) fabrication [4].

Optical layout of the LITI system is similar to the laser marking system equipped with a beam scanning device. Besides the laser, marking devices are typically consist of beam expander, $f-\theta$ lens, and Galvano-mirror. The Galvano-mirror controls the laser beam direction before the beam is focused by an $f-\theta$ lens. The most widely applied laser in the marking industry is diodepumped solid-state laser with TEM $_{00}$ mode. Recently, the single mode fiber laser is gaining popularity due to its advantages such as simple operation, small volume, high power, and long lifetime.

In a laser marking system, the amount of evaporated material depends on the incident laser intensity and energy. A $\mathrm{TEM}_{00}$ mode laser or single mode fiber laser

*Corresponding author: jhyi@yu.ac.kr has a Gaussian spatial intensity distribution. The Gaussian beam has the functional form $I_{0} \exp \left(-r^{2} / 2 \omega_{0}^{2}\right)$, where $r$ is the distance from the center of the beam and $\omega_{0}$ is the beam radius. When the Gaussian beam is incident on the target surface, the amount of evaporated material from the target substrate becomes a function of distance from beam center [5]. Therefore non-uniformity of evaporated material from the surface degrades the precision and the sharpness of the marked letters or sign. The problem caused by the Gaussian beam is also an important issue in the LITI system because the system also uses laser energy for local heating of the donor film to detach organic layer [4].

In some laser marking devices, beam shaping optics are used to deliver a flat-top beam to enhance marking quality. In such devices, the beam shaping optics is usually placed between the beam expander and the Galvanomirror. Various optics such as lens set [6], micro lens array [7], diffractive optical elements [8], random phase plate [9], light guiding plate [10], and acousto-optic modulator (AOM) [4] have been used for beam shaping. The lens set method is frequently employed in commercially developed marking systems. Spherical aberrations of spherical lenses [6] or specially designed aspheric lenses [11] were used for beam shaping. The micro-lens array method is another well developed method, and 
it requires delicate design procedure depending on the marking laser beam conditions. The random phase plate or diffractive optical element beam shaping device also requires a lithography process for fabrication.

Acousto-optic method has been used in the LITI system [4]. The beam is usually scanned by a Galvanomirror. In LITI, an organic film layer is detached from donor film. The irradiated laser energy is converted to heat in the heat absorption layer of the donor film. It is known that the laser beam shape which has more energy at both sides of the beam track is desirable for clean and sharp patterning [12]. To get the profile, the direction of the laser beam is dithered in high frequency by an AOM transversely to the beam scanning direction.

In this work, we devised beam shaping optics using hollow optical fiber (HOF) to replace the bulky and expensive AOM. In the course of propagating through the $\mathrm{HOF}$, the laser beam redistributes its energy evenly along the circular clad. When the ring-shaped beam is focused and scanned on the donor film surface, the energy distribution becomes similar to the result obtained when the dithered beam by an AOM was used. Beam shaping optics using light guiding was also employed in the display industry for the laser annealing of polysilicon [10]. Compared to the other beam shaping methods, the device using light guiding is less affected by the spatial fluctuation of the input beam. Generation of a hollow beam by HOF was also tried to guide cold atoms by another group [13].

\section{EXPERIMENT AND RESULTS}

To compare beam shapes before and after propagating the hollow fiber, we sent a He-Ne laser beam to a HOF. The laser beam was coupled into the clad of the HOF by focusing the laser beam with a plano-convex lens of 5 -cm focal length. The schematic experiment setup is shown in Fig. 1(a). The silica fiber used in the experiment had $200 \mu \mathrm{m}$ inner diameter and $355 \mu \mathrm{m}$ outer diameter (Polymicro Technology). The transmitted beam was collimated by another plano-convex lens of $5-\mathrm{cm}$ focal length. The beam profiles were captured by a CCD camera and the image was analyzed by beam analysis software (Spiricon). To capture the beam profile at the fiber exit, the collimated beam was imaged onto the CCD surface by a focusing lens which has the same focal length as the collimation lens.

As shown in Fig. 1(b), the Gaussian beam before the fiber was transformed to a ring-shaped pattern after transmission through the fiber. The beam diameter at the exit surface of the fiber became larger than the spot size of the input beam at the fiber entrance surface because the fiber clad diameter was larger than the input beam spot size. The figure indicates that the laser energy was redistributed into a lot of propagation modes of

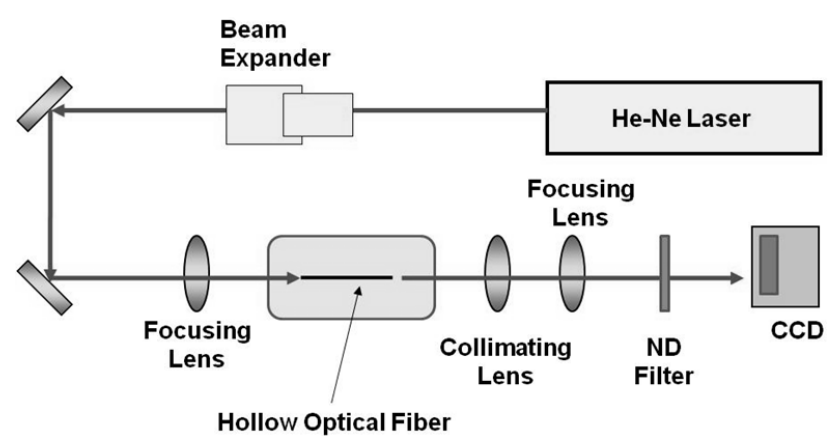

(a)

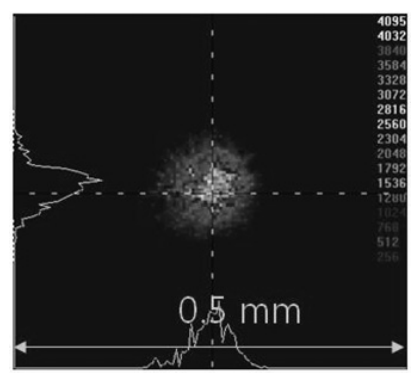

Before Hollow Optical Fiber

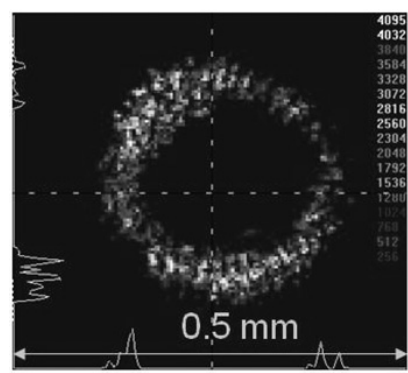

After Hollow Optical Fiber (b)

FIG. 1. (a) Optical layout of the experimental setup for beam shaping using hollow optical fiber (CCD: charge coupled device, ND: neutral density). (b) The beam profiles before and after hollow fiber propagation.

the fiber. As the number of modes was very large, the laser energy showed almost even distribution along the circular silica clad cross-section.

There should be appropriate length of the fiber needed for the laser beam to form a uniform ring-shaped pattern. To estimate the length, we tried non-sequential ray tracing simulation using commercial software (Light Tools). As the clad of the fiber has much larger dimension than the propagating laser wavelength, diffraction was not considered. In the simulation, a Gaussian beam of the He-Ne laser was generated by placing a point source at the focus of a parabolic mirror. The spatial profile of the ray intensity reflected from the parabolic mirror (dotted line) became quite similar to the Gaussian distribution (solid line) as can be seen in Fig. 2(a). The evolution of ray intensity profiles while propagating through the hollow fiber were simulated as a function of propagation distances. We found that the intensity profile became almost independent to the fiber length when the length was longer than about $5 \mathrm{~cm}$, as can be seen in Fig. 2(b) (e). It is also found that the output was unaffected by the laser beam spot position on the fiber input surface.

When the Gaussian beam was focused on the hollow core of $\mathrm{HOF}$, part of the propagating rays were leaked out of the fiber to the air. Part of the focused rays trans- 


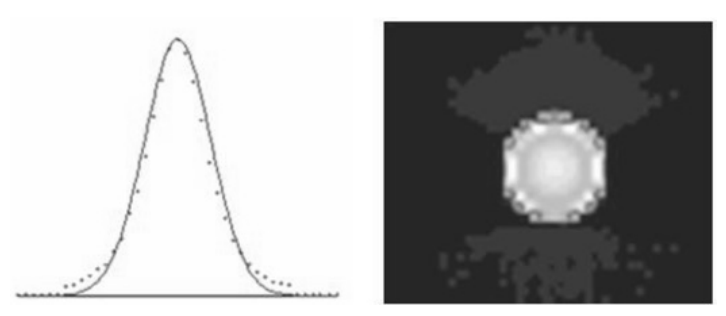

(a)
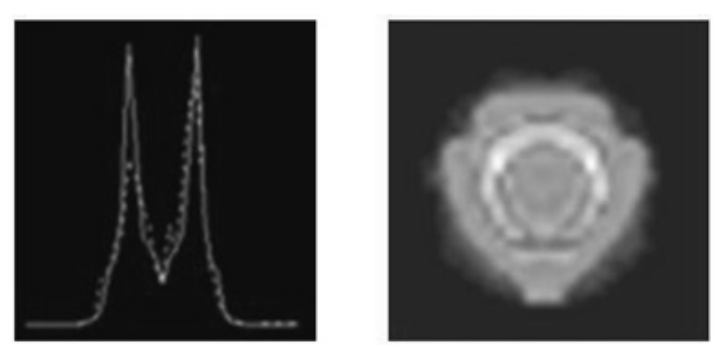

(b) $\mathrm{L}=25 \mathrm{~mm}$
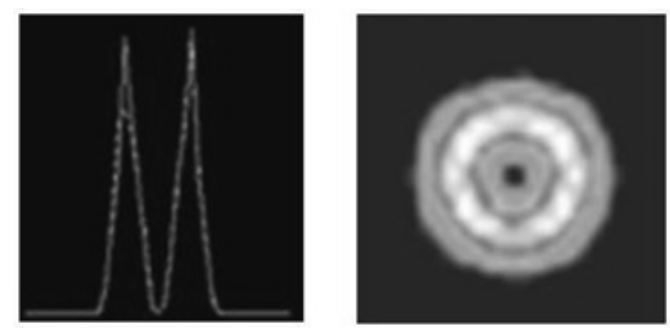

(c) $\mathrm{L}=50 \mathrm{~mm}$
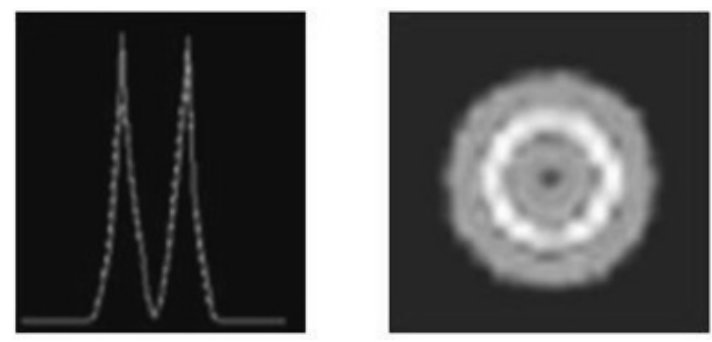

(d) $\mathrm{L}=80 \mathrm{~mm}$
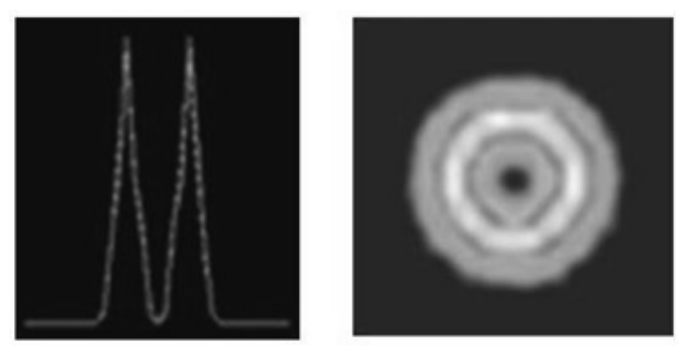

(e) $\mathrm{L}=100 \mathrm{~mm}$

FIG. 2. (a) The simulated Gaussian beam (dotted line) and calculated Gaussian beam (solid line). Simulated results of transformed beam profiles after the hollow fiber propagation for several lengths of the hollow fiber, L, are shown. (b) $\mathrm{L}=25 \mathrm{~mm}$, (c) $\mathrm{L}=50 \mathrm{~mm}$, (d) $\mathrm{L}=80 \mathrm{~mm}$, (e) $\mathrm{L}=100 \mathrm{~mm}$.

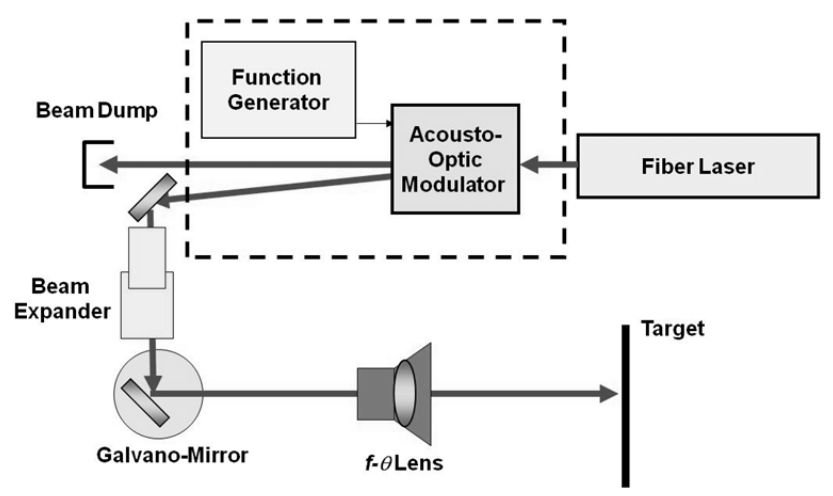

FIG. 3. Schematic layout of laser thermal printing system. Acousto-optic modulator dithers the beam in high frequency for beam shaping.

mitted inside the silica clad and the transmitted rays had larger angle of incidence than the critical angle for total internal reflection. After the rays were focused on the silica clad of the fiber, the rays could be confined inside the clad in the simulation. However, in the experiment, part of the beam coupled into the clad leaked out of the fiber due to surface contacts at fiber holding mounts. The scattering from the fiber cylinder surface due to dust or micro defect also attenuated the beam. Outer clad with lower index of refraction is required to hold the power inside the inner clad for practical application.

The calculated output intensity distribution shown in Fig. 2(c) is more uniform than the experimentally obtained result shown in Fig 1(b). In the experiment, the surface roughness of fiber end surface at exit side affected the output beam intensity distribution considerably. To enhance the beam profile, the fiber ends were polished until a fine surface was obtained. Before polishing procedure, the inner part of hollow core was filled with resin. When polishing of the fiber ends was finished, the resin was removed by immersing the fiber for several hours in acetone. The polished ends were examined by using a microscope. As the surface crosssection was very small, good polishing over the entire surface was hard to obtain. Cleaving of the fiber was also tried. However the end surfaces became worse than polished results as the process required delicate skill.

Figure 3 shows the schematic experimental setup for LITI. Usually Nd:YAG laser has been applied in the system [11] while we propose here to use cw fiber laser considering high stability and small volume of the laser. The fiber laser (IPG) has polarized output and it is focused onto the clad of a 5 -cm long HOF by a planoconvex lens of 5 -cm focal length. The fiber laser has wavelength of 1060 1070 nm and beam diameter of $4 \mathrm{~mm}$. The fiber laser beam is deflected by the AOM. The diffraction efficiency of the AOM was around $80 \%$. The deflected beam is directed to a beam expander with 


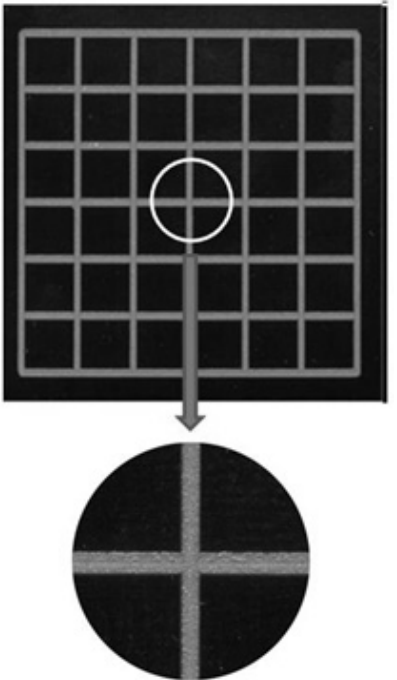

(a)

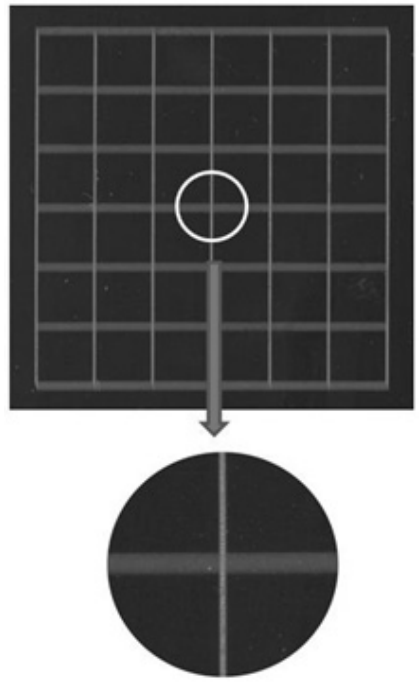

(b)
FIG. 4. The lattice are marked on photographic sheets by either (a) ring-shaped beam after hollow fiber propagation or (b) dithered beam by acousto-optic modulator. Part of the lines are expanded to show details of the pattern.

magnification of 2 . The beam expander is used to get narrower spot size at the donor film. The expanded beam is sent to galvano-mirrors. The reflected beam from galvano-mirrors is focused on the donor film by an $f-\theta$ lens of $160 \mathrm{~mm}$ focal length. An arbitrary two dimensional pixel pattern designed by the computer is printed on the flat panel. The deflection angle is modulated with high frequency following the signal fed into the AOM driver from the function generator.

The laser beam distribution at the donor film surface is determined by the waveform and frequency of signal from the function generator. Typical waveforms such as sine, saw tooth, or square waves were tried. Lines of the lattices shown in Fig. 4 were experimentally obtained by using a beam modulated by AOM method (Fig. 4(a)) or shaped by a HOF (Fig. 4(b)). When we tested a $\mathrm{HOF}$, we replaced $\mathrm{AOM}$ at the dotted area shown in Fig. 3 by HOF. A lens $(\mathrm{f}=5 \mathrm{~cm})$ condenses the fiber laser beam onto the entrance surface of hollow fiber (outer diameter $=355 \mu \mathrm{m}$, inner diameter $=200 \mu \mathrm{m}$ ). The coupling and collimation setup is similar to the set up shown in Fig. 1(a). The collimated beam is directed to the beam expander. The result shows that the track obtained from AOM method has the more sharp edge compared with the track obtained from the HOF method. Asymmetrical shape of the focused beam trace in HOF case is caused by non-uniform distribution of energy along the ring-shaped pattern due to fiber exit surface defect.

When the uniform ring-shaped beam is scanned in the $y$ axis direction, as illustrated in Fig. 5(a), each

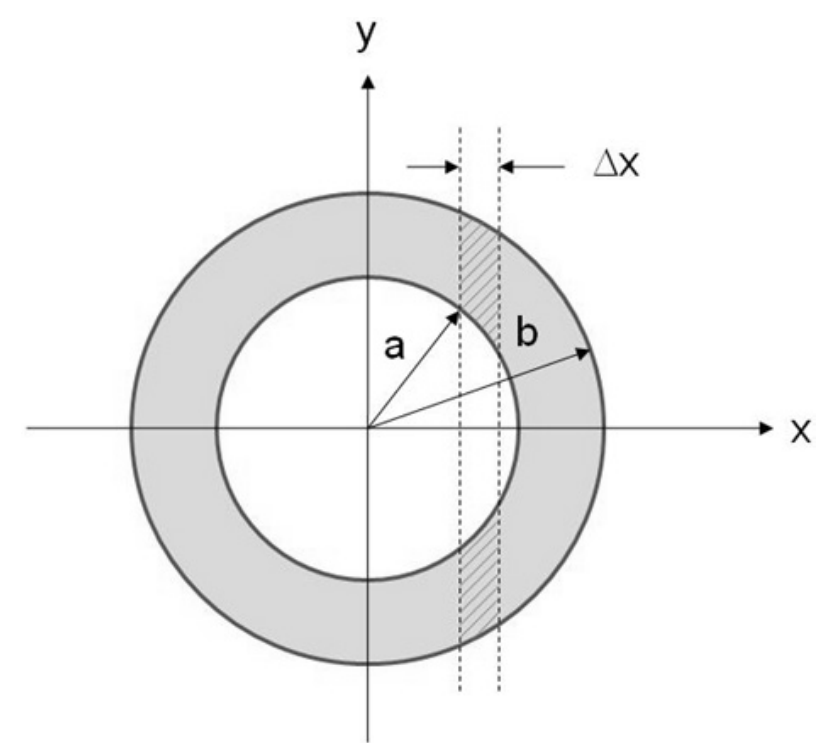

(a)

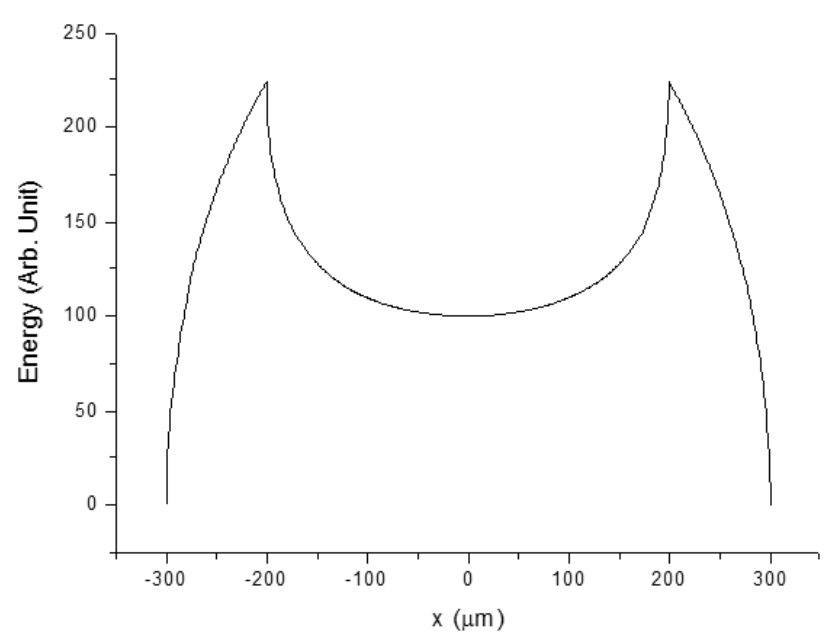

(b)

FIG. 5. (a) The cross-section view of the ring-shaped beam. The beam is scanned along $y$-axis. (b) The distribution of the laser energy incident on a point on $x$-axis is drawn as a function of $x$ when the ring-shaped beam is scanned along $y$-axis.

point on $x$-axis would experience different total laser energy. If the energy is uniformly distributed on a ringshaped cross-section, the total energy received within the band $\Delta x$ on the $x$-axis would be proportional to the hatched area shown in Fig. 5(a). Assuming uniform distribution of the laser beam on a circular ring, the amount of energy received at a point $x$ from the center of the beam, $I(x)$, can be approximated as,

$$
I(x) \propto 2\left(\sqrt{a^{2}-x^{2}}-\sqrt{b^{2}-x^{2}}\right) \Delta x
$$

In the equation, $a$ is outer radius and $b$ is inner radius of the ring-shaped beam as shown in Fig. 5(a). 


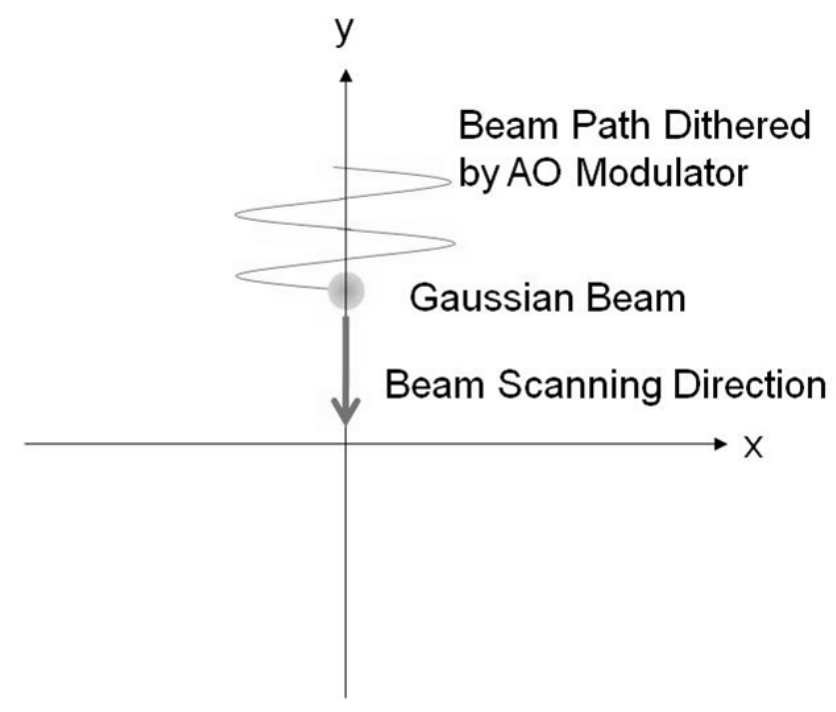

(a)
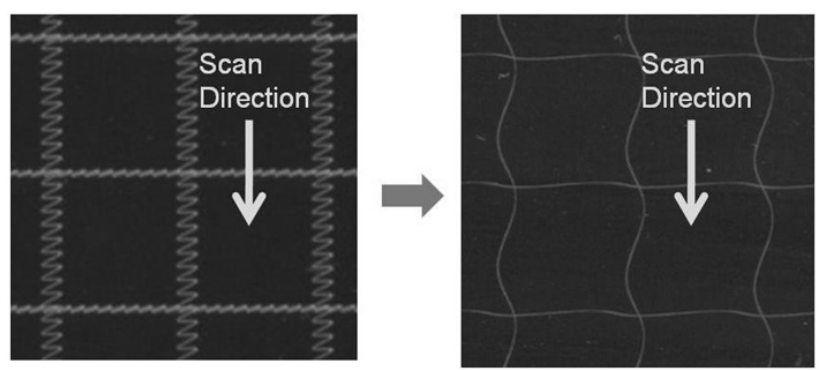

(b)

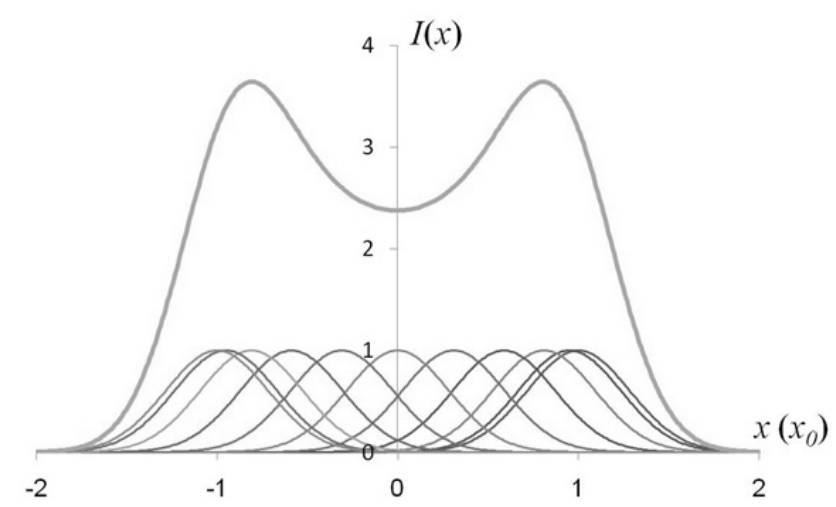

(c)

FIG. 6. (a) Trajectory of dithered beam. (b) The recorded trace of beam when the scan speed is high (left) and slow (right) (c) The laser beam energy distribution as a function $x$ is drawn. Several profiles of moving Gaussian beam (thin solid lines) are shown in temporal interval of $\mathrm{T} / 20$ (T: period) and total energy distribution is denoted as thick line.

The calculated laser energy as function of $x$ on axis, transverse to the scanning direction, is shown in Fig. 5(b). The profile shows peaks at both sides of the beam trace.
In the AOM method, scanning laser beam is dithered in a way illustrated in Fig. 6(a). The beam path depends on the scan speed as well as dithering frequency. Left figure in Fig. 6(b) has 10 times slower scan speed than right figure in Fig. 6(b). When the scan speed is very slow and the dithering frequency is very high, pitch of the trace becomes negligible. In this case, the incident energy distribution on the surface along the $x$-axis can be approximated as follows. Dithered beam trace is nearly moving parallel to the $x$-axis and the moving speed of the beam is a function of distance $x$ from the origin. Suppose the dithering follows a sine wave, then the incident energy distribution on the x-axis during half of the dithering period, $T / 2$, can be approximated as,

$$
I(x) \propto k \int_{0}^{T / 2} \exp \left[-\frac{\left(x-x_{0} \cos \frac{2 \pi}{T} t\right)^{2}}{\omega_{0}^{2}}\right] d t .
$$

In the equation, $k$ is a proportional constant, $x_{0}$ is dithering amplitude, and $\omega_{0}$ is the waist of the Gaussian beam. Figure 6(c) shows the calculated result when $\omega_{0}=0.4 x_{0}$. The profile also shows peaks at both sides. The width of each peak becomes narrower as the ratio $\omega_{0} / x_{0}$ moves to smaller value. The intensity at edge sharply drops to zero when the ratio approaches zero. The profile is very similar to the profile shown in Fig. 5(b) which is obtained by scanning a ring-shaped beam. The profile close to the one obtained from the delicate dithering process was simply made by using a ring-shaped beam.

\section{CONCLUSION}

A beam shaping device suitable for laser induced thermal printing system was devised. The device reshapes the energy distribution of a Gaussian beam to a ringshaped beam by sending the Gaussian beam through the hollow optical fiber (HOF). When the focused ringshaped beam is scanned on a plane following a linear track, the accumulated energy distribution showed peaks at both sides of the track. The distribution was compared to the result obtained when the linearly scanned Gaussian beam was dithered transversely by an acousto-optic modulator. We found that the results obtained by two methods are very similar after comparison of the energy distribution. The method using $\mathrm{HOF}$ as a passive optic gives a simple and cheap means of beam shaping. In case when the $\mathrm{HOF}$ is spliced with the end of the fiber laser directly, the hollow fiber device should provide a more robust beam shaper. This result should be also applicable to a laser marking system. 


\section{ACKNOWLEDGMENT}

This research was supported by the Yeungnam University research grants in 2007.

\section{REFERENCES}

1. T. Arai, N. Morosawa, Y. Hiromasu, K. Hidaka, T. Nakayama, A. Makita, M. Toyota, N. Hayashi, Y. Yoshimura, A. Sato, K. Namekawa, Y. Inagaki, N. Umezu, and K. Tatsuki, "Micro silicon technology for active matrix OLED display," in Proc. Society for Information Display 2007 International Symposium Digest (2007), paper 41.2.

2. K. Matsuo, K. Hanawa, T. Hirano, T. Sasaoka, and T. Urabe, "LIPS (Laser-Induced Patternwise Sublimation) technology for manufacturing large-sized OLED displays," in Proc. Society for Information Display 2007 International Symposium Digest (2007), paper OLED2-1.

3. T. Hirano, K. Matsuo, K. Kohinata, K. Hanawa, T. Matsumi, E. Matsuda, R. Matsuura, T. Ishibashi, A. Yoshida, and T. Sasaoka, "Novel laser transfer technology for manufacturing large-sized OLED displays," in Proc. Society for Information Display 2007 International Symposium Digest (2007), paper 53.2.

4. S. T. Lee, J. Y. Lee, M. H. Kim, M. C. Suh, T. M. Kang, Y. J. Choi, J. Y. Park, J. H. Kwon, and H. K. Chung, "A new patterning method for full-color polymer light-emitting devices: laser induced thermal imaging (LITI)," in Proc. Society for Information Display 2002 International Symposium Digest (2002), paper 21.3.

5. J. F. Ready, Industrial Applications of Lasers (Second edition, Academic Press Sandiego, 1997), Chapter 12.

6. I. A. Al-Saidi, "Using a simple method: conversion of a Gaussian laser beam into a uniform beam," Opt. and Laser Technology 33, 75-79 (2001).

7. C. Kopp, L. Ravel, and P. Meyrueis, "Efficient beamshaper homogenizer design combining diffractive optical elements, microlens array and random phase plate," J. Opt. A, Pure Appl. Opt. 1, 398-403 (1999).

8. Q. Li , H. Gao, Y. Dong, Z. Shen, and Q. Wang, "Investigation of diffractive optical element for shaping a Gaussian beam into a ring-shaped pattern," Opt. Las. Tech. 30, 511-514 (1998).

9. Y. Kato, K. Mima, N. Miyanaga, S. Arinaga, Y. Kitagawa, M. Nakatsuka, and C. Yamanaka, "Random phasing of high-power lasers for uniform target acceleration and plasma-instability supression," Phys. Rev. Lett. 53, 1057-1060 (1984).

10. T. Okamoto, K. Morokawa, A. Sono, Y. Sato, and J. Nishimae, "Development of line-shaped optical system for green laser annealing used in the manufacture of low-temperature poly-Si thin-film transistors," Appl. Opt. 45, 4709-4714 (2006).

11. J. A. Hoffnagle and C. M. Jefferson, "Design and performance of a refractive optical system that converts a gaussian to a flat-top beam," Appl. Opt. 39, 5408-5499 (2000).

12. S. T. Lee, B. D. Chin, M. H. Kim, T. M. Kang, M. W. Song, J. H. Lee, H. D. Kim, H. K. Chung, M. B. Wolk, E. Bellmann, J. P. Baetzold, S. Lamansky, V. Savvateev, T. R. Hoffend Jr., J. S. Staral, R. R. Roberts, and Y. Li, "A novel patterning method for fullcolor organic light-emitting devices: laser induced thermal imaging (LITI)," in Proc. Society for Information Display 2004 International Symposium Digest (2004), paper 29.3.

13. J. Yin, Y. Zhu, W. Jhe, and Y. Wang, "Atom guiding and cooling in a dark hollow laser beam," Phys. Rev. A 58, 509-513 (1998). 